\title{
Mechthild Veil \\ Der Beitrag der Kindererziehung für die Alterssicherung von Frauen und Eltern. Drei Vorschläge zur Diskussion
}

Die Rentenversicherung ist wieder in der Diskussion. Nachdem die Auseinandersetzungen nach der Rentenreform ' 92 zunächst einmal verstummt waren, brechen alte und neue Kontroversen über Grundlagen und Zielsetzung der Alterssicherung auf. Denn die enge, auf die Systematik der Rentenversicherung begrenzte Sichtweise des Rentenreformgesetzes '92 (RRG '92) hatte langfristige Probleme der Alterssicherung nicht in Angriff genommen. Bereits im Vorfeld des RRG '92 wurden Fragen zur zukünftigen Entwicklung der Alterssicherung auf die Finanzierung einer ungünstigen demographischen Entwicklung reduziert und der gesellschaftliche Wandel in anderen Bereichen wie z. B. auf dem Arbeitsmarkt (jobless growth), in den Familienstrukturen und vor allem im Geschlechterverhältnis ausgegrenzt.

Bisher unbeantwortet ist, wie eine eigenständige Sicherung von Frauen auszugestalten sei. ${ }^{1}$ Vorschläge hierzu liegen seit Beginn der 8 oer Jahre vor, zum Beispiel in dem Modell von Krupp u. a., ${ }^{2}$ das später von Gabriele Rolf und Gert Wagner zum "Voll Eigenständigen System « (VES) weiterentwickelt wurde. ${ }^{3}$ Statt dessen wird die Zukunft der gesetzlichen Rentenversicherung (GRV) zunehmend unter dem Aspekt der Familienpolitik und des Familienlastenausgleichs diskutiert, eine Perspektive, die in dem Elternrenten-Modell von Jürgen Borchert ${ }^{4}$ konzentriert zum Ausdruck kommt. Nach diesen Vorstellungen wird die Rentenversicherung auf eine generative Grundlage gestellt, die - so meine These - auf einem falsch verstandenen Generationenvertrag beruht.

Familie, Kinder und Generationenfolge scheinen der Themenkomplex zu sein, der zukünftig die Rentendiskussion am stärksten beeinflussen wird. In den Vordergrund geraten Diskussionsströme, die bisher weder von den Akteuren der Rentenpolitik noch von der professionellen Sozialbürokratie zur Kenntnis genommen wurden: das heikle Thema der generativen Grundlagen der Rentenversicherung. 'Diskutiert wird der Zusammenhang von Alterssicherung und Familie und die Bedeutung, die Kinder für die eigene Altersvorsorge haben. Zugespitzt geht es um die Frage, ob es sich mehr lohnt, in die eigene Berufstätigkeit oder in Kinder zu investieren. Dabei gehen die mit dem Elternrenten-Modell transportierten Vorstellungen von einem militanten Familienverständnis in der Alterssicherung aus, das Frauen als eigenständiges Rechtssubjekt nicht geiten läßt.

Um so anerkennenswerter ist es, wenn $B$. Krrsc $h^{6}$ die eigenständige Sicherung von Frauen unter dem Gesichtspunkt ihrer individuellen Rechtsposition noch einmal

I Vgl. Benno Kırsch: Gerechtıgkeıt und „Entscheidungshılfen «- dıe Situatıon von Frauen ım Rentenrecht seit der Rentenreform 1992, in: Kritısche Justız, 2/1995, S. 173-188.

2 Hans-Jurgen Krupp, Heınz Galler, Heinz Grohmann, Rıchard Hauser, Gert Wagner (Hg.): Alternatıven der Rentenreform '84. Frankfurt/New York $198 \mathrm{r}$.

3 Vgl. Gabriele Rolf/Gert Wagner: Ziele, Konzepte und Detailausgestaltung des "Voll-Eigenstandıgen Systems « der Altersvorsorge, in: Sozıaler Fortschritt, 12/1992, S. 28 I-291.

4 Jurgen Borchert: Innenweltzerstorung, Frankfurt a. M. 1989; ders., Renten vor dem Absturz, Frankfurt a. M. 1993.

s Vgl. Thomas Ebert: Familienfundamentalismus und Alterssicherung, in: WSI Mitteilungen, 6/1995, S. 373 .

6 Kirsch (Fn. 1) 
thematisiert und hierfür aktuelle Analysen vorstellt und diskutiert. ${ }^{7} \mathrm{Zu}$ Recht kritisiert er, daß die Rentenversicherung beinahe ausschließlich auf der Erwerbsarbeit beruht und Kindererziehung zu wenig honoriert. Das fördert den Anschein, »die höheren Renten der Männer seien allein deren Verdienst ${ }^{8}{ }^{8}$ In seinen Schlußfolgerungen jedoch greift $B$. Kirsch auf ein Verständnis vom Generationenvertrag zurück, das ich nicht teile. Auch er spricht, im Sinne J. Borcherts, von der "nötigen Kinderzahl" als Grundlage der Renten und davon, daß Kinderlose "im Alter durch die Beitragszahler, die von anderen aufgezogen wurden «, 9 abgesichert werden.

Im folgenden soll nun der Versuch unternommen werden, anknüpfend an B. Kirsch auf die neue Strömung des Familienfundamentalismus in der Alterssicherung,$^{10}$ d. h. konkret auf das Elternrenten-Modell von J. Borchert einzugehen, und dieses mit zwei weiteren Modeflen und Zielvorstellungen zu konfrontieren: den Reformvorstellungen des Verbandes Deutscher Rentenversicherungsträger (VDR) und dem Voll-Eigenständigen System (VES) von Krupp u. a., weitergeführt von Rolf/Wagner.

\section{Die Rolle des Bundesverfassungsgerichts als Motor für Rentenreformen}

In den letzten Jahren ist die Rentenpolitik der Bundesregierung durch Initiativen unterschiedlicher Akteure in Zugzwang geraten, die Tempo und Richtung einer zukünftigen Reform der Alterssicherung prägen. Das am 25. Juli 199r verkündete Renten-Überleitungsgesetz (RÜG)" setzte die bundesdeutschen Vorschriften zur gesetzlichen Rentenversicherung mit Wirkung vom 1. Januar 1992 auch in den neuen Bundesländern in Kraft. Mit einer Entschließung von Bundestag und Bundesrat vom 21. Juni $199 \mathrm{I}^{12}$ hatte sich die Bundesregierung zu einer Reform der Alterssicherung von Frauen verpflichtet, indem die Zeit bis zum Auslaufen der Bestandsschutzregelungen ${ }^{13}$ (1996) im Rahmen des RÜG genutzt werden soll, die Alterssicherung von Frauen in der leistungsbezogenen Rentenversicherung zu verbessern. Kernpunkte der avisierten Veränderungen sind: Verbesserung der Absicherung aus Zeiten der Kindererziehung und der Pflege, Ausbau der eigenständigen Rentenansprüche von Frauen, Lösung des Problems ihrer Altersarmut.

Desweiteren mahnt das Bundesverfassungsgericht (BVerfG) seit längerem Reformen zur Alterssicherung von Frauen und zum Familienlastenausgleich an. Zum Steuerrecht forderte das BVerfG in zwei Entscheidungen vom 29. Mai $1990^{14}$ und vom 12. Juni 1990, 's daß bei der Einkommensbesteuerung ein Betrag in Höhe des Exi-

7 Mechthıld Veil u.a. (Hg.): Am modernen Frauenleben vorbe.. Verliererınnen und Gerwinnerınnen der Rentenreform ' 92 , Berlin 1992; Jutta Allmendınger u. a.: Arbeitsleben und Lebensarbeitsentlohnung, in: Claudia Gather u.a. (Hg.): Frauen-Alterssicherung, Berlın 1992; Gabriele Rolf: Ideologiekritik am Rentenrecht und ein Reformvorschlag zur ergenstandigen Alterssicherung von Frauen, in: Gather u.a. (Hg.), ebd.

8 Kirsch (Fn. 1), S. 186.

9 Ebd., S. 583.

10 Ebert (Fn. 5), S. 365

If Gesetz zur Herstellung der Rechtseinheit in der gesetzlichen Renten- und Unfallversicherung (BGBI. I, S. 1606).

12 BT-Drucks. $12 / 837$

13 Mit den Bestandsschutzregelungen wurden niedrige ehemalıge DDR-Renten durch den sog. Sozıalzuschlag vorubergehend aufgestockt, damit Rentner durch die Rentenuberleitung nicht schlechter gestellt werden als vorher. Die Bestandsschutzregelungen laufen I 996 aus, wodurch ene nicht unerhebliche Gruppe v. a. von Rentnerınnen in die Sozialhılfe gedrangt wird.

${ }_{14}$ BVerfGE 82, 7 .

15 BVerfGE 82, 12. 
stenzminimums der Familie steuerfrei bleiben muß. Zur Rentenversicherung hat das BVerfG in dem sog. "Kindererziehungszeiten-Urteil « vom 7.7.1992 ${ }^{16}$ festgestellt, $\mathrm{da} ß \mathrm{das}$ geltende Rentenrecht Kindererziehende benachteiligt und gefordert, »... den Mangel des Rentenversicherungssystems, der in den durch Kindererziehung bedingten Nachteilen bei der Altersversorgung liegt, in weiterem Umfang als bisher auszugleichen. « ${ }^{17}$ Obgleich sich das BVerfG auf keine konkreten Vorschläge festlegte, erschien ihm der weitere Ausbau von Kindererziehungszeiten als geeignetes Mittel.

Dem Spruch des BVerfG lagen zwei Verfassungsbeschwerden von Frauen zugrunde, die gegen den unzureichenden Stellenwert der Kindererziehung im Kinderleistungsgesetz (KLG) und im Hinterbliebenen- und Erziehungszeitengesetz (HEZG) klagten und forderten, Kindererziehungszeiten "als eine den monetären Beiträgen gleichwertige Beitragsform» in der gesetzlichen Rentenversicherung zu berücksichtigen. Die Mißachtung der generativen Leistungen widerspräche Art. 6 Abs. I GG und würde kinderlose Paare auf Kosten kinderreicher Familien bevorzugen und ihre Renten durch die Erziehungsleistungen anderer sichern. Folgerichtig forderten die Klägerinnen eine stärkere Berücksichtigung des Familienlastenausgleichs im Rentenrecht, entsprechend dem Kinderleistungsgesetz (KLG) und der Hinterbliebenenrente. ${ }^{18}$

Das BVerfG hatte die Beschwerden zurückgewiesen, jedoch eine großzügige Anerkennung von Kindererziehungszeiten angemahnt. Da sich aus dem Verfassungsauftrag - einen wirksamen Familienlastenausgleich zu schaffen - keine konkreten Forderungen ableiten lassen, wie die staatliche Familienförderung in den Teilsystemen zu verwirklichen sei, hatte das BVerfG dem Gesetzgeber Gestaltungsfreiheit eingeräumt.

Das BVerfG ging in seiner Begründung ausdrücklich auf die bestandssichernde Bedeutung der Kindererziehung für die Altersversorgung ein. "Denn die als Generationenvertrag ausgestaltete Rentenversicherung läßt sich ohne die nachrückende Generation nicht aufrecht erhalten. «' Einen bemerkenswerten Vorstoß unternahm das BVerfG in der Frage, wie die Reformvorschläge finanziert werden könnten: durch die Modifikation der Hinterbliebenenrente, »... das Grundgesetz (läßt) Raum für eine Änderung der Hinterbliebenenversorgung mit dem Ziel, bei Witwen- und Witwerrenten stärker auf die Dauer der Ehe sowie darauf abzustellen, ob der überlebende Ehepartner durch Kindererziehung oder Pflegeleistungen in der Familie am Erwerb einer eigenen Altersversorgung gehindert war. ${ }^{20}$ Das BVerfG plädierte für mehr Familienpolitik im Rentenrecht.

In der Auseinandersetzung um die Anerkennung von Kindererziehungszeiten ging es den Klägerinnen um die Besserstellung kinderreicher Familien gegenüber Kinderlosen und nicht um eine bessere Absicherung von Frauen in einer eigenständigen Sicherung. In den Begründungszusammenhängen wurde nicht mehr, wie noch in den 7oer und 8oer Jahren, die Alterssicherung von Frauen gegenüber Männern thematisiert, ${ }^{21}$ sondern der Schutz kinderreicher Familien durch eine Verbesserung des

16 BVerfGE 87, 1 .

17 BVerfGE 87, I (Leitsatz 2).

18 Vgl. Mechthild Veil: Ene familienfreundliche oder frauenfeindliche Entscheidung? Das BVerfG vom 7.Julı 1992 zur Anrechnung von Kindererziehungszetten im Rentenrecht, in: Streıt. Femınıstische Rechtszeitschrift. $1-2 / 1993$, S. 63-74

19 BVerfGE 87, 1, S. 70.

20 BVerfGE 87, 1, S. 6i f.

21 Stellvertretend fur viele: Annelies Kohleıss: Frauenrechte in der gesetzlichen Rentenversıcherung, in: Ute Gerhard/Alice Schwarzer/Vera Slupik (Hg.): Auf Kosten der Frauen. Frauenrechte im Sozıalstaat, Weinheim und Basel 1988 , S. $117^{-1} 7^{2}$. 
Familienlastenausgleichs in der Alterssicherung. Dieser Perspektivwechsel, mit dem die Alterssicherung von ihren generativen Grundlagen her neu aufgerollt wird, kennzeichnet die aktuelle Diskussion zur Rentenreform.

Die Signalwirkung, die von der BVerfG Entscheidung ausgeht, ist ambivalent. Einerseits wird die Altersarmut von Frauen, die durch lange Zeiten der Kindererziehung keine ausreichenden Rentenansprüche aufbauen können, thematisiert. Die nun auch verfassungsrechtlich unterstützte Ausweitung der Kindererziehungszeiten wird die Rentensituation von Frauen verbessern helfen. Inwieweit die erweiterten Erzichungszeiten als Bausteine für eine eigenständige Sicherung von Frauen ausgebaut werden können, hängt davon ab, ob sie zukünftig additiv zu einer Erwerbstätigkeit gewährt werden. ${ }^{22}$ Denn nur dann wird es Frauen und Männern möglich, Beruf und Familie zu vereinbaren. Dazu hat sich das BVerfG jedoch nicht geäuBert.

\section{Das Elternrenten-Modell: Lastenausgleich für Mütter}

Das Eltern-Renten-Modell wurde bereits Anfang der $80 e r$ Jahre von J. Borchert, Richter am Hessischen Landessozialgericht, entwickelt. J. Borcherts immer wieder variierte Grundthese ist einfach: Das Steuer- und Sozialversicherungssystem, insbesondere die Rentenversicherung, ist eltern- und kinderfeindlich. Das auf die Erwerbsarbeit ausgerichtete Rentensystem erkennt nur unzureichend Erziehungsleistungen an und bevorzugt Alleinstehende oder kinderlose Ehen mit zwei Einkommen gegenüber Familien mit Kindern. ${ }^{23}$

Kindererziehung soll für die spätere Rentenhöhe den gleichen Rang erhalten wie die Zahlung von Beiträgen. Dazu wird das gesamte verfügbare Rentenvolumen zur Hälfte als Erwerbsrenten und zur Hälfte als Elternrenten aufgeteilt. Für alle Erwerbstätigen (Beitragszahler) halbiert sich bei gleichbleibender Beitragszahlung der Rentenanspruch, so daß eine Rente nach 45 Versicherungsjahren und mit durchschnittlichem Gehalt von derzeit rd. 2000,- DM auf 1000,- DM zusammenschrumpft. Eine kontinuierliche Berufstätigkeit in Vollzeit würde demnach zu einer Alterssicherung führen, die gerade rd. I 50,- DM über dem Sozialhilfesatz liegt. Die andere Hälfte des Rentenvolumens stände dann für eine beitragsfreie Elternrente zur Verfügung. Eltern erhalten danach für jedes Kind (Obergrenze bei vier Kindern) einen Rentenanspruch von ca. 700,-- DM (1993). Damit führt die Erziehung von zwei Kindern ohne eigene Beitragszahlung zu einer gleich hohen Rente, wie ein $45 j$ jähriges Erwerbsleben mit durchschnittlichem Gehalt.

Die Elternrente wird auf die Partner so verteilt, daß der nichterwerbstätige Erziehende (meist die Frau) die Elternrente erhält. Sind beide berufstärig, dann reduziert sich die Elternrente reziprok mit der Hälfte der beitragspflichtigen Rentenansprüche. „Hat der Vater z. B. einen monetär erworbenen Rentenanspruch in Höhe von 1000,- DM und die Mutter einen solchen von 500,- DM, so erhält sie von der Elternrente zwei Drittel und er ein Drittel. $\aleph^{24}$ Bei Alleinerziehenden wird die besondere Belastung berücksichtigt, sie haben Anspruch auf die ungeteilte Elternrente.

\footnotetext{
22 Nach dem HEZG werden Kindererziehungszeiten nur berucksıchtigt, wenn sie Versıcherungslucken fullen. Beı eınem Zusammentreffen mit anderen versıcherungsrechtlıchen Zeıten konnen niedrige Rentenanspruche durch die Babyrente lediglich auf dieses Niveau aufgestockt werden.

23 Vgl. Borchert: Renten vor dem Absturz $\left(F_{n}, 4\right)$, S. $269 \mathrm{ff}$.

24 Ebd., S. 271.
} 
Mit der Elternrente entfällt die Hinterbliebenenrente und damit jeder Rentenanspruch für kinderlose Hausfrauen mit der Begründung: Die Solidargemeinschaft der Versicherten soll nicht für die private Lebensplanung einer »unbegründeten «, da kinderlosen, Hausfrauenexistenz finanziell aufkommen. ${ }^{25}$

Das Modell führt zu gewaltigen Umverteilungen der Rentenansprüche von Kinderlosen zu kinderreichen Familien. Vorteile gegenüber dem geltenden Recht haben erst Familien ab zwei bis drei Kindern mit nur einem Verdiener. Erst dann, wenn die Halbierung der lohnbezogenen Rentenansprüche nicht stärker zu Buche schlägt als die zusätzlichen kindbezogenen Ansprüche einbringen, ergeben sich Verbesserungen. ${ }^{26}$ Alle anderen Familien sowie Paare ohne Kinder, Alleinstehende und Singles werden schlechter gestellt. ${ }^{27}$

Der Verdienst J. Borcherts liegt darin, daß er die bekannten und immer wieder kritisierten Ungerechtigkeiten des Rentensystems gegenüber Frauen und Familien cindringlich brandmarkt. Er nennt Armutsdaten älterer kinderreicher Frauen und zeigt auf, daß Kinder der wesentliche Grund für Niedrigrenten an Frauen sind.

Die Nachteile jedoch überwiegen. Das Elternrenten-Modell richtet sich gegen die Berufstätigkeit von Müttern und gegen die Kinderlosigkeit von Frauen und Männern. Je weniger Frauen mit Kindern sich beruflich engagieren, desto größer sind die Vorteile, die sie aus dem dualen Rentenmodell ziehen.

Die Anreize, die das duale System der Elternrente schafft, stehen in diametralem Gegensatz zum geltenden Recht. Geht das geltende Rentenrecht von dem Individuum und seiner Stellung auf dem Arbeitsmarkt, unabhängig vom Familienstand aus, so das duale System von der Familie mit Kindern. Normiert das geltende Recht den kontinuierlich und in Vollzeit Berufstätigen (männliche Normalbiographie) auf Kosten von Frauen mit Kindern, die mit Teilzeitarbeit und Berufsunterbrechungen versuchen, Beruf und Familie zu vereinbaren, so setzt das Modell von J. Borchert als Norm die traditionelle Zwei-Kind-Familie, die den Mann zur Berufsarbeit und die Frau zur Hausfrauenexistenz verpflichtet - auf Kosten aller anderen erwerbstätigen Männer und Frauen. Liegt die Diskriminierung von Frauen im geltenden Recht darin, daß Familienarbeit kaum berücksichtigt wird, so daß mit steigender Kinderzahl die Renten an Frauen sinken, so diskriminiert umgekehrt die Elternrente die Erwerbstätigkeit von Frauen mit und ohne Kinder.

Das Elternrenten-Modell beruht auf einem alten Mißverständnis über die Rolle des Generationenvertrages in der gesetzlichen Rentenversicherung. Die aus der katholischen Soziallehre stammende Konzeption geht von dem Bild einer Gesellschaft aus, die auf familialer Subsidiarität beruht. ${ }^{28}$ Die Rentenversicherung wird als Institutionalisierung einer gesellschaftlichen Entwicklung gesehen, die von der vorindustriellen Drei-Generationen-Familie zur »kollektiven Großfamilie der familialen Gesellschaft « geführt habe. Die Solidargemeinschaft der Versicherten, die an die Stelle der solidarischen Großfamilie getreten sei, erwirtschaftet die Renten nicht nur durch Erwerbsarbeit, sondern auch durch die »Aufzucht « von Kindern.

Die Anhänger eines so verstandenen Drei-Generationen-Vertrages beklagen, daß mit der großen Rentenreform 1957 unter Adenauer die Renten nicht in diesem Sinne umgestellt worden seien. Sie können sich mit ihrer Kritik auf Oswald von Nell-

25 Ebd., S. 271.

26 Vgl. Ebert (Fn. 5), S. 371

27 Zur Kurzdarstellung und Diskussion der vorliegenden Rentenmodelle vgl. Deutscher Gewerkschaftsbund - Bundesvorstand - Abtellung Frauenpolitik ( $\mathrm{Hg}$.): Alterssicherung von Frawen - Probleme und Losungsansatze, Dusseldorf $1 / 1995$.

28 A Annlich dazu vgl. Frank Nullmeier/Friedbert W. Rub: Erschopfung des Sozıalversicherungsprinzıps? Gesetzliche Rentenversicherung und sozialstaaticher Republikanismus, in: Leviathan. Sonderheft $14 / 1994$, S. 73 . 
Breuning und Ferdinand Oeter, zwei »Väter« der dynamischen Rente, beziehen, die das duale Modell unterstützten, um den Kardinalfehler von 1957 zu korrigieren. ${ }^{29}$ Übersehen wird dabei, daß die Rentenreform von 1957 auf die veränderten Familienstrukturen und die damit verbundene nachlassende familiale Subsidiarität durchaus reagierte, indem die Alterssicherung von einer minimalen Grundsicherung (im Kapitaldeckungsverfahren), die noch auf ergänzende Familienhilfe angewiesen war, auf individuelle Ansprüche aus den je individuellen Erwerbsbiographien (im Umlageverfahren) umgestellt wurde. Erst damit wurde die Emanzipation der Eltern von ihren Kindern möglich.

Die dynamische Rente stellt einen neuen Generationenvertrag her. Dieser beruht nicht mehr auf Familienbeziehungen, mit einem privilegierten Zugriff der Älteren auf die Produktivkraft ihrer Kinder, sondern auf der Teilhabe der bereits aus dem Berufsleben ausgeschiedenen Generation an der wirtschaftlichen Gesamtentwicklung. Dies wurde erreicht, indem die Rentenberechnung und die jährlichen Rentenanpassungen dynamisiert wurden. Mit der Umstellung von der Bruttolohn- auf die Nettolohnanpasssung hat das RRG '92 diesen Zusammenhang noch verstärkt. ${ }^{30}$

Die Krux der Rentenreform 1957 liegt nicht in der Loslösung der Alterssicherung von familialer Unterstützung, sondern darin, daß sie einseitig auf Erwerbsarbeit zentriert wurde und damit automatisch Frauen schlechter stellt, die für die Kindererziehung und Pflegeleistungen häufig eine Erwerbstätigkeit unterbrechen oder aufgeben. Die Solidarität der Solidargemeinschaft bezieht sich auch heute nur unzureichend auf die Lebenszusammenhänge von Frauen.

Es ist schon erstaunlich, daß diese überzogenen demographischen Argumente von $J$. Borchert u. a. so attraktiv werden konnten. Drückt doch die immer wieder betonte Bedeutung des "Nachwuchses « für eine Rentenversicherung im Umlageverfahren nicht einfach die simple Tatsache aus, daß jede staatliche und gesellschaftliche Ordnung auf eine sich selbst reproduzierende Bevölkerung für ihren Fortbestand angewiesen ist? J. Borcherts Popularität liegt darin, daß er Schwachstellen des Molochs Sozialstaat, die regierungsamtlich tabu sind, aggressiv und schnoddrig diskutiert. So dramatisiert er die Bevölkerungsentwicklung zu einem Krieg der Generationen, indem er aufzeigt, »... daß immer mehr der ursprünglich für die Jugend bestimmten Mittel (des Sozialbudgets, M. V.) zu den Alten umgeleitet werden. « ${ }^{31}$ Damit habe sich »die relative Stellung der Jugend seit einer Reihe von Jahren immer mehr verschlechtert, während die der Alten sich verbesserte. “32 Durch »eine egoistische Anpassung der Verteilungssysteme an die Bedürfnisse der älteren Generation « 33 verspiele die Sozialpolitik solange die Zukunft der Jugend, bis diese die Verteilungsungerechtigkeiten gewaltsam zu lösen versuche.

J. Borchert weiß, daß ohne Krisenszenarien weder grundlegende Reformen noch Utopien denkbar sind. Das wissen auch die GRÜNEN. Als eine Partei, die Zukunftsfragen thematisiert und eher die Interessen Jugendlicher und Menschen im mittleren Alter als die der Rentner repräsentiert, sind sie für Borcherts Argumente aufgeschlossen.

29 Vgl. Borchert, Renten vor dem Absturz (Fn. 4), S. 274.

30 Vgl. Nulimerer/Rub (Fn. 28), S. 64

31 Borchert (Fn. 4), 1989, S. 86.

32 Ebd., S. 89 .

33 Ebd., S. 90 

Äquivalenzprinzip der Renten

Im Gegensatz zum Elternrenten-Modell stehen dic Vorstellungen des Verbandes Deutscher Rentenversicherungsträger (VDR). Der VDR hält im wesentlichen strikt an dem Äquivalenzprinzip der Rentenversicherung fest, d. h. Renten sind das Ergebnis eigener Beitragszahlung und spiegeln im Alter den durch Erwerbsarbeit erworbenen Lebensstandard wider. Ansprüche aus beitragslosen Zeiten wie der Kindererziehung gelten als versicherungsfremde Leistungen. Der VDR fordert, daß diese vom Staat und nicht von der Versichertengemeinschaft zu finanzieren seien. ${ }^{31}$

Die strenge Unterscheidung zwischen Aufgaben des Staates (Familienlastenausgleich) und Aufgaben der Solidargemeinschaft begründet $F$. Ruland mit den unterschiedlichen Personengruppen, die jeweils zahlen und Leistungen empfangen. Mit Versicherungsbeiträgen dürften nicht Leistungen finanziert werden, die an Personen außerhalb der Solidargemeinschaft gehen. Denn das konstituierende Element der gesetzlichen Rentenversicherung sei die Typisierung von Risiken, die sozial abgesichert werden. Eine so verstandene Solidarität sei gegenüber der Solidarität in der Bevölkerung eingeschränkt. Die Erfüllung des Generationenvertrages sei demzufolge eine gesellschaftliche Aufgabe, die nicht der gesetzlichen Rentenversicherung aufgebürdet werden könne. Dies geschehe jedoch, wenn der Staat Sozialpolitik betreibr, zum Beispiel Kindererziehungszeiten (als Teil des Familienlastenausgleichs) in der Rentenversicherung ausbaut und die Kosten nur einem Teil der Bürger aufhalse. ${ }^{35}$ F. Ruland tritt für eine genauere Differenzierung der Finanzierungsströme zwischen Staat und GRV ein.

Einseitig thematisiert der VDR Finanzierungsprobleme, die auf die Altersstruktur zurückzuführen sind, obgleich die Rentenversicherung gerade durch die Massenarbeitslosigkeit und die Vielzahl der ungeschützten Arbeitsverhältnisse zunehmend vor große Belastungen gestellt wird. Da der VDR sich nicht zur Arbeitsmarktpolitik der Bundesregierung äußert, kann er sich auch nicht produktiv, d. h. mit eigenen Vorschlägen einmischen. Nur so ist es zu verstehen, daß, unabhängig von empirischen Befunden, der Verband an einem globalisierenden Modell des Rentners festhält und eher dazu tendiert, "schlechte" Risiken auszusteuern als durch Umverteilungsmaßnahmen die Privilegien des männlichen Normalarbeiters anzutasten. ${ }^{36}$

Nach wie vor spielt Teilzeitarbeit in Reformvorhaben keine Rolle, weder die versicherungspflichtige noch die versicherungsfreie. Der Verband äußert sich zur geringfügigen Beschäftigung zurückhaltend und lehnt die Einführung einer Versicherungspflicht »ab der ersten Arbeitsstunde« ab. Da diese nur zu minimalen Rentenansprüchen führe, könne sie der Forderung nach Einführung einer Mindestrente und nach sozialem Ausgleich mehr Gehör verschaffen - eine Entwicklung, die der VDR jedoch zu bremsen versucht. ${ }^{37}$

Aus dieser Grundposition heraus ist es dem VDR nicht möglich, Träger umfassender Reformen zu sein und gesellschaftliche Perspektiven für eine Neuorientierung der sozialen Sicherung zu eröffnen. Der VDR beschränkt sich auf Vorschläge, Kindererziehungszeiten (allerdings steuerfinanziert) zusätzlich zu anderen Beitragszeiten aus der Erwerbsarbeit zu berücksichtigen.

34 Vgl. Franz Ruland: Versıcherungsfremde Leistungen in der gesetzhchen Rentenversicherung, in: Deutsche Rentenversicherung, I/1995, S. 28-38.

35 Ebd., S. 30 .

$36 \mathrm{Vgl}$. Margarete Landenberger: Die Beschaftıgungsverantwortung der Rentenversıcherung. Berlin 1990, S. 66 und S. $102 \mathrm{f}$.

37 Ebd., S. 65 . 

Frauen

Das Voll-Eigenständige System (VES) ${ }^{38}$ hat den Anspruch, eine eigenständige Sicherung für Frauen zu schaffen, die durch Änderungen im bestehenden Rentenrecht erreicht wird. Die Autoren kritisieren am bestehenden System, daß es die Nichterwerbstätigkeit verheirateter Frauen subventioniert und z. B. bei Scheidung einer lang andauernden Hausfrauenehe der Gang zum Sozialamt vorprogrammiert sei. Das sei in einer modernen Alterssicherung nicht mehr zu verantworten. Mit ihrem Modell sollen demgegenüber Anreize für eine verstärkte Erwerbsbeteiligung verheirateter Frauen und für die Vereinbarkeit von Beruf und Familie als Aufgabe beider Geschlechter geschaffen werden.

Diese Ziele werden vom Voll-Eigenständigen System durch zwei Instrumente erreicht: durch eine Mindestbeitragspflicht für alle Personen und durch ein Splitting der während der Ehe gemeinsam erworbenen Rentenanwartschaften. ${ }^{39}$ Ausgangspunkt der Überlegungen ist, daß alle Erwachsenen ab dem $2 \mathrm{r}$. Lebensjahr versichert sind und über eine eigene Alterssicherung verfügen. Dies soll dadurch erreicht werden, daß ab dem 2 r. Lebensjahr alle Bewohner der Bundesrepublik pflichtversichert sind. Für Nichterwerbstätige zahlt die Person oder Institution die Beiträge, die für den laufenden Lebensunterhalt aufkommt (Mindestbeiträge in Höhe von $75 \%$ des Durchschnittsgehaltes aller Versicherten). Erwerbstätige entrichten wie bisher ihre Beiträge aus Erwerbseinkommen, die niedriger ausfallen können, da Hinterbliebenenrenten entfallen.

Im einzelnen heißt das:

Für Personen, die durch Arbeitslosigkeit, Krankheit oder Ausbildung an einer Berufstätigkeit gehindert sind, übernimmt die zuständige Institution die Beitragszahlung (Mindestbeitrag), z. B. die Bundesanstalt für Arbeit. Für Kindererziehende zahlt der Staat den Mindestbeitrag für drei Jahre. Für nichterwerbstätige Hausfrauen müßte der Ehemann zahlen. Für Alleinerziehende und kinderreiche Familien mit niedrigem Einkommen ist eine steuerfinanzierte Subventionierung vorgesehen. Nur für Sozialhilfeempfänger sieht das Modell keine Pflichtversicherung vor, so daß dieser Personenkreis auch im Alter auf unterstützende Sozialhilfe angewiesen sein wird.

Das Modell führt zu einer Individualisierung von Rentenansprüchen und zu einer ausreichenden Grundabsicherung. Verheiratete (Haus-)Frauen werden nicht mehr auf vom Mann abgeleitete Renten wie die Hinterbliebenenrente verwiesen, sondern Männer und Frauen verfügen nach diesem Modell über eigene Rentenkonten. Durch das Rentensplitting, das bei Rentenbeginn des ersten Partners durchgeführt wird, sind beide Ehepartner auf gleichem Niveau abgesichert.

Die Vorteile des Voll-Eigenständigen Systems liegen auf der Hand: Es sollen eigenständige Rentenanwartschaften für Frauen aufgebaut, ein Beitrag zum Abbau der Altersarmut von Frauen geleistet und die Berufsorientierung von Frauen sowie die Vereinbarkeit von Beruf und Familie gefördert werden. Gegenüber der normativen Position des geltenden Rechts, das die Nicht-Erwerbstätigkeit verheirateter Frauen honoriert und Kindererziehung nicht gleichzeitig zu einer Berufstätigkeit anerkennt, fordert das Voll-Eigenständige System die additive Anrechnung von Kindererziehungszeiten, um Beruf und Familie vereinbaren zu können.

$38 \mathrm{Vgl}$. Rolf/Wagner (Fn. 3) - Das Modell wurde modifizıert von der SPD-Bundestagsfraktıon als "Eıgenstandige Alterssicherung der Frauen " ubernommen. Zur Kritsk an dem SPD-Modell vgl. Ruland: Anmerkungen zum Konzept der Projektarbestsgruppe "Eıgenstandige Alterssıcherung der Frau* der SPD-Bundestagsfraktion, in: Deutsche Rentenversicherung, 10/1994, S. 670-681.

39 Rolf/Wagner (Fn 3), S. 284 . 
Das Voll-Eigenständige System und die Elternrente sind zwei Modelle, die mit völlig unterschiedlichen Intentionen die reproduktiven Arbeiten von Frauen für die Rente anerkennen. Im Elternrenten-Modell geschieht dies in Abgrenzung und auf Kosten von Erwerbstätigen, im Voll-Eigenständigen System dagegen durch einen sanften finanziellen Druck zur Aufnahme einer Berufstätigkeit. Das Voll-Eigenständige System geht von dem Leitbild einer Ehe mit zwei Berufstätigen aus und erkennt die wachsende Bedeutung der Erwerbsarbeit für Frauen an.

Die kritischen, noch zu diskutierenden Punkte beziehen sich auf folgendes:

Das Modell setzt Zeiten der Vollbeschäftigung voraus. Bei einer hohen Arbeitslosigkeit werden nach dem Voll-Eigenständigen System einkommensschwache Familien mit nur einem Verdiener stärker als bisher belastet, was sozialpolitisch wohl kaum zu vertreten ist. Da aus den Erwerbseinkommen auch Beiträge für den nichterwerbstätigen.Partner finanziert werden müssen, führt das Voll-Eigenständige System desweiteren nicht nur zu einer Umverteilung von Männer- zu Frauenrenten - was kein Fehler wäre --, sondern auch zu einer höheren Belastung von jungen, noch im Erwerbsleben stehenden Personen gegenüber der Generation der Rentner. ${ }^{\circ}$ Solch eine Entwicklung ist äußerst problematisch und wird bereits bestehende Spaltungslinien zwischen Jungen und Alten, an die Borchert anknüpft, verschärfen.

\section{Schlußbemerkungen}

Mit dem Elternrenten-Modell, den Vorstellungen des VDR und dem Voll-Eigenständigen System sind die wesentlichen Reformvorschläge zur Bedeutung der Kindererziehung für die Alterssicherung umrissen. Sie unterscheiden sich vordergründig in der Frage ihrer Finanzierung: beim VDR über Steuern, beim Voll-Eigenständigen System über Beiträge des unterhaltspflichtigen Ehepartners und in den ersten drei Jahren Kindererziehung über Steuern, bei der Elternrente über Beiträge aller Erwerbstätigen. Die Art der Finanzierung führt zu völlig unterschiedlichen Umverteilungsprozessen: zwischen Männern und Frauen, Kinderlosen und Kinderreichen, zwischen den Generationen oder zwischen der gesetzlichen Rentenversicherung und dem Staat.

Hinter den Modellen und Zielvorstellungen stehen unterschiedliche Wertevorstellungen über die Familie und die Rolle der Frau. Anhänger des Drei-GenerationenVertrages, wie J. Borchert, F. Oeter und O. von Nell-Breuning, kritisieren an der dynamischen Rente, daß sie den Zusammenhang von familialem Unterhalt und Sozialleistung zerstört. Sie streben an, die Familie als Produktionseinheit mit einer strikten Arbeitsteilung zwischen der Erziehenden und dem Familienernährer zu erhalten. Eine Individualisierung von Rentenansprüchen lehnen sie deshalb genauso ab wie die Individualisierungsprozesse in der Gesellschaft, durch die Frauen nicht mehr unbedingt nur Mütter und Männer nicht mehr ausschließlich Väter sind.

Welche der Diskussionsströme - Individualisierung von Rechtsansprüchen und Stärkung der Erwerbsposition von Frauen wie im Voll-Eigenständigen System oder Ausbau des Familienlastenausgleichs und der Familienpolitik wie im ElternrentenModell, wodurch die Gruppe der erwerbstätigen Frauen an Gewicht verliert - sich langfristig in der Rentenpolitik durchsetzen wird, ist noch nicht abzusehen. Forderungen zum Ausbau individueller Rentenansprüche im Sinne der Vereinbarkeit von Beruf und Familie werden von der Politik der Europäischen Gemeinschaft unter-

40 Vgl. DGB (Fn. 27), S. 18 . 
stützt. Sie ist Teil der EU-Politik zur Verwirklichung der Chancengleichheit von Frauen im Arbeitsleben. ${ }^{41}$ "Die EG-Gemeinschaftsorgane haben im Zuge ihrer Politik der Gleichbehandlung der Frau in der sozialen Sicherheit das Prinzip der Individualisierung von Rechtsansprüchen (individualisation of entitlements) in den verschiedenen Systemen der sozialen Sicherheit besonders betont. « $^{42}$ Demgegenüber ist die deutsche Diskussion stark auf Familie und den längst überfälligen Ausbau des Familienlastenausgleichs konzentriert. Inwieweit die stärkere Berücksichtigung familienpolitischer Belange in der Rentenversicherung erfolgt, ist und bleibt ungewiß.

Eine zukünftige Rentenreform müßte von der Individualisierung von Rechtsansprüchen auf der Basis einer ausreichenden Grundsicherung ausgehen und das Ziel einer eigenständigen Sicherung für Frauen im Sinne des Voll-Eigenständigen Systems verfolgen. Dabei kann auf das BVerfG verwiesen werden, das in seinem »Kindererziehungszeiten-Urteil« eine großzügige Anerkennung von Kindererziehungszeiten fordert und ausdrücklich feststellt, daß »die deutlich höhere Betroffenheit von Frauen« bei der Kindererzichung eine Pflicht für den Gesetzgeber auslöst, »auf eine Angleichung der Lebensverhältnisse von Frauen und Männern hinzuwirken. «43 Für dieses Ziel sind Rahmenbedingungen notwendig, die es Männern und Frauen erlauben, Beruf und Familie miteinander zu vereinbaren. Zu dicsen Rahmenbedingungen gehört auch die Art und Weise, wie die Alterssicherung ausgestaltet ist.

\section{Joachim Perels}

\section{Kontinuität im Kampf um der Menschen Rechte Heinrich Hannover zum 70. Geburtstag}

Heinrich Hannover, geboren 1925 , ist es nicht an der Wiege gesungen worden, zu einem der profiliertesten linken Anwälte der Bundesrepublik zu werden. Als junger Soldat nimmt er am Krieg Hitlers teil. Er sieht ihn als Kampf fürs Vaterland an. Sein Vater, ein Chefarzt, gehört zu den Anhängern des Regimes. Nach dem frühen Tod seiner Eltern ganz auf sich gestellt, macht Hannover nach einem halbjährlichen Schnellkurs im Anschluß an die I I. Klasse Abitur. Seine Selbsttätigkeit bildet sich früh aus. ${ }^{1}$

Als Jura-Student in einer Verbindung organisiert, begreift er sich als cher unpolitischen Juristen. Zwei Schlüsselerfahrungen aber verändern ihn. Seit 1954 ist er in Bremen als Anwalt zugelassen. In der Verbindung, der er angehört, entsteht ein Konflikt um die Frage, ob die Vertretung von Kriegsdienstverweigerern, die sich dem sogenannten Dienst fürs Vaterland entziehen, mit den Zielen der studentischen Vereinigung vereinbar ist. Hannover, durch das Erleben der militärischen Tötungsmaschinerie der Nazis zum Kriegsgegner geworden, bricht mit seiner Verbindung, die anwaltlichen Beistand für Kriegsdienstverweigerer mehrheitlich für verbands-

41 Vgl. Maxmmılian Fuchs: Vereınbarkeit von Familie und Beruf als Gestaltungsaufgabe der Rentenversıcherung, in: Deutsche Rentenversicherung, 10/1994, S.659.

42 Ebd., S. 665 .

43 BVerfGE 87, I, S. 63.

1 Vgl. auch zum folgenden Heike Mundzeck, "... sch wurde lıeber Kınderbuchautor seın .... Heınrich Hannover im Gesprach, in: „Eine Zeıt, in der die Falschen verurtelt werden«. Leben fur ein demokratısches Recht. Heinrich Hannover zum 60. Geburtstag, Hamburg 1985, S. 123 ff. 